\title{
PAISAJES CULTURALES Y PROYECTO TERRITORIAL: un balance de treinta años de experiencia
}

\author{
Joaquín Sabaté Bel \\ Universitat Politècnica de Catalunya
}

\section{Paz Benito del Pozo}

Universidad de León

\section{RESUMEN}

En este artículo se desarrolla una valoración sintética de la consideración que han ido ganando los "paisajes del trabajo" ${ }^{1}$ en España durante los últimos treinta años, del marco en el que se han desenvuelto y de las principales intervenciones sobre los mismos. ${ }^{2}$ Se quiere destacar, en primer lugar, cómo algunos agentes han construido un "estado de opinión", contribuyendo a un creciente interés por dicho patrimonio. Esto incluye a profesionales,

\footnotetext{
${ }^{1}$ Aunque el objeto de atención sea esencialmente, aunque no en exclusiva, el patrimonio industrial, creemos interesante referirnos al patrimonio minero, agrícola, de infraestructuras...(a todo lo relacionado con el trabajo) y por ello la expresión "paisajes del trabajo" podría ser más útil que la de paisaje industrial. No se hará referencia en cambio a una acepción más tradicional de patrimonio (arqueológico, histórico, artístico, arquitectónico...). De hecho la definición de paisajes del trabajo coincidiría con la de paisaje cultural que hemos utilizado en diversos estudios, basada en los trabajos de Carl Sauer. Aunque goza de reconocimiento oficial, todavía hoy paisaje cultural constituye un término poco común para un concepto reconocimiento oficial, todavía hoy paisaje cultural constituye un término poco común para un concepto
relativamente opaco. Proponemos una definición algo más sencilla: paisaje cultural es un ámbito geográfico asociado a un evento, a una actividad o a un personaje con dimensión histórica, que contiene valores estéticos y culturales. O dicho de una manera menos ortodoxa, pero más sencilla y hermosa, paisaje cultural es la huella del trabajo sobre el territorio, algo así como un memorial al trabajador desconocido. Esta temática la hemos abordado en textos como: "Paisajes culturales en Cataluña: el eje patrimonial del río Llobregat" y "De la preservación del patrimonio a la ordenación del paisaje" en El paisaje y la gestión del territorio. Criterios paisajísticos en la ordenación del territorio y el urbanismo. Diputación de Barcelona. Barcelona, 2006.

${ }^{2}$ No pretendemos construir un "estado del arte" exhaustivo, simplemente destacar aquellos elementos más relevantes.
} 
departamentos universitarios y centros de investigación, pero asimismo se considera la labor de difusión a través de publicaciones, congresos y museos. Del marco en el que se han desenvuelto cabe referirse a algunas normas que han tenido una clara incidencia en este campo.

Finalmente se pretende verificar como en bien pocos años hemos ido desarrollando instrumentos de intervención cada vez más complejos, comprometidos y de mayor escala, desde los primeros inventarios, catálogos y trabajos de documentación; a las intervenciones aisladas, de recuperación y reutilización de edificios singulares; a los planes y programas donde se integra el patrimonio industrial (del trabajo en general) en propuestas coordinadas de mayor ambición y finalmente, a proyectos territoriales, donde dicho patrimonio constituye el eje de la intervención y puede devenir motor de desarrollo local.

En el límite cabría aspirar a que el patrimonio industrial, el del trabajo en general, juegue un papel equivalente en cualquier proyecto o plan territorial, al que ha alcanzado el patrimonio natural; que cultura y natura se conviertan en ejes de reflexión y medida de nuestras intervenciones sobre el territorio.

\section{ABSTRACT}

This article evaluates the consideration that have gained the "work landscapes" in Spain during the last thirty years, the context in which they have operated and some major interventions. We want to emphasize how some agents have built a "state of mind," contributing to a growing interest in this heritage. They include professionals, university departments and research centers. The dissemination through publications, conferences and museums is also relevant, and also certain laws have a clear impact on this field.

We also verify how in few years we have developed intervention tools to operate over complex and large scale areas. These tools evolve from early inventories, catalogs and documentation works, to single interventions for the 
recovery and reuse of landmark buildings; to plans and programs that integrate the industrial heritage, and finally to regional projects, where this heritage is the backbone of the intervention which may become a local development engine. We aim that work or industrial heritage, should play in any proposal at urban or territorial scale, the same role that natural heritage has reached, We aim that culture and nature become main axis in our territorial interventions.

\section{EL CRECIENTE INTERÉS POR EL PATRIMONIO DEL TRABAJO}

\subsection{La aportación de los estudiosos}

El interés social por los restos industriales (fábricas, colonias textiles, mineras, puertos...) arranca precisamente del proceso de desindustrialización en los años 70 y de las propuestas para reactivar los territorios afectados.

Una primera reacción procede de ámbitos académicos y profesionales diversos, y sostiene que los edificios industriales pueden ofrecer valores que aconsejan su conservación y reutilización, ${ }^{3}$ o que los territorios industriales en desuso, forman parte de nuestro patrimonio cultural. ${ }^{4}$ Algunos geógrafos destacan las oportunidades urbanísticas asociadas al aprovechamiento de las piezas industriales abandonadas en grandes ciudades, ${ }^{5}$ o profundizan en la relación entre patrimonio industrial y desarrollo local, ${ }^{6}$ entre patrimonio cultural

\footnotetext{
3 Capel, H. (1996 ). "La rehabilitación y el uso del patrimonio histórico industrial", Documents d'Anàlisi Geogràfica, nº 29, pp. 19-50.

4 Ortega, J. (1998). "El patrimonio territorial. El territorio como recurso cultural y económico", Ciudades, $\mathrm{n}^{\circ} 4$, Instituto de Urbanística de la Universidad de Valladolid, pp. 3348.

5 Pardo, C.; Olivera, A. (1992), "'Les friches industrielles à Madrid: opportunités urbanistiques et réutilisation du sol urbain", Revue Belgue de Géographie, n¹16 anne, 1-4, Fascicule 51.

${ }^{6}$ Rodríguez, F. (1992). "El patrimonio industrial histórico como recurso para el desarrollo local", en Ábaco. Revista de Ciencias Sociales, $2^{\circ}$ época, n¹, Oviedo, pp. 71-78.

Troitiño, M.A. (1998). "Patrimonio arquitectónico, cultura y territorio", Ciudades, n 4, Instituto de Urbanística de la Universidad de Valladolid, pp. 95-104.
} 
y ordenación del territorio, ${ }^{7}$ o en la aplicación de los vestigios industriales al desarrollo territorial, lo que incluye la recuperación de paisajes amenazados por la destrucción de sus elementos más singulares. ${ }^{8} \mathrm{~A}$ medida que se hacen evidentes los efectos de la crisis y el declive industrial madura, toma cuerpo una corriente de opinión sensible con los restos industriales, que cristaliza en la década de los 90 con propuestas de intervención fundadas en su valor testimonial y material, así como en su potencial para servir de reclamo cultural, para convertirse en producto turístico, y para actuar como factor de revitalización socioeconómica y recuperación de la identidad de territorios en crisis. ${ }^{9}$ Algunos años antes se formulan incluso unas primeras propuestas de un eje patrimonial basado en la explicación del impulso inicial, auge y fracaso de la industrialización, y en la reutilización del patrimonio del trabajo como fundamento de un nuevo desarrollo local. ${ }^{10}$

\subsection{La labor pionera de los centros de investigación}

La Universidad se suma al estudio y defensa del paisaje del trabajo con numerosas investigaciones y cursos, donde destaca la importancia y potencial de dicho patrimonio, a la vez que debate metodologías de intervención. En general, predomina un concepto del patrimonio industrial que se asocia con la arqueología, la historia de la técnica, el arte, e incluso la etnografía, si bien el factor territorial empieza a adquirir una presencia cada vez más notable.

\footnotetext{
7 Bielza de Ory, V.; De Miguel, R. (1997). "El patrimonio cultural, componente de la ordenación del territorio", Revista Aragonesa de Administración Pública, n 10, pp. 185-ss.

${ }^{8}$ Benito, P. (1997). "Dinamización del territorio y patrimonio industrial", Polígonos, n 7, pp. 123-131.

Fernández, A. (1999). "El Valle del Samuño: argumentos para su consideración como patrimonio histórico de la minería asturiana", Ería, n50, pp. 279-289.

Feliú, A. (1998). "El patrimonio industrial, localizaciones, regeneraciones: una nueva geografía”, Ábaco, n¹9, pp. 71-80.

${ }^{10}$ Sabaté, J.; Schuster, J.M. (ed) Designing the Llobregat Corridor. Cultural Landscape and Regional Development. Projectant l'eix del Llobregat. Paisatge cultural i desenvolupament regional. Universidad Politécnica de Cataluña y Massachusetts Institute of Technology. Barcelona, 2001.
} 
Como en otros países, las iniciativas de puesta en valor de los paisajes del trabajo han tenido en España un triple origen: la reflexión universitaria; la labor de alguna administración especialmente sensibilizada; y el trabajo de agentes locales, amantes de un territorio en el que pretenden valorizar su patrimonio. $Y$ afortunadamente estos tres grupos han tendido a converger y a sumar sus esfuerzos en repetidas ocasiones. La labor de diversos departamentos universitarios en Valencia, León, Barcelona o Sevilla, entre otros, es bien remarcable; como lo es la del Instituto Andaluz del Patrimonio Histórico, y de manera excepcional la de INCUNA, impulsando una notable labor de investigación y difusión del patrimonio industrial asturiano, y del español en general.

A modo de ejemplo, cabe señalar la labor realizada en el seno del Departamento de Urbanismo y Ordenación del Territorio de la Universidad Politécnica de Cataluña. A finales de los ochenta arrancan unos trabajos de investigación que llevan a la presentación de una tesis doctoral que analiza un tramo de apenas veinte kilómetros del río Llobregat. ${ }^{11}$ Este curso fluvial atesora un patrimonio extraordinario, formado por catorce colonias industriales.

Casi en paralelo, la Diputación de Barcelona encarga al mismo departamento la redacción de un plan urbanístico del Parque Agrario, vieja reclamación de agricultores locales y de algunos técnicos de la propia Diputación. Su ámbito constituye un territorio de notable productividad y extensión en medio de un entorno metropolitano muy poblado y sometido a un deterioro considerable, derivado en buena medida de servidumbres metropolitanas y de su condición peri-urbana. Esto implica un proceso acelerado de pérdida de calidad ambiental, disminución de cultivos, abandono de la actividad profesional. Resultaba imprescindible un proyecto que, atendiendo a la identidad de este espacio agrario, planteara un modelo de futuro.

${ }^{11}$ El Sistema de Colònies Tèxtils del Baix Berguedà. Gènesi i Revaloració" Tesis doctoral de Pere Vall, dirigida por Joaquín Sabaté. De manera resumida se recoge en el libro De colònies tèxtils a Parc de les Colònies. El Sistema de Colònies Tèxtils del Baix Berguedà, Gènesi i revaloració. Editorial Marcombo S.A. Barcelona, 1999. 
Este fue el objetivo fundamental del Plan Especial: proyectar la estructura de un territorio rural, descubriendo y actualizando la que informó su construcción (división del suelo, alteración del relieve, organización de los caminos de tierra y de agua y establecimiento de las construcciones), así como reconocer el equivalente en el territorio agrícola, de los sistemas de espacios libres, dotaciones, accesos y servicios, que sustentan los tejidos urbanos. El Parque Agrario constituye hoy una iniciativa pionera estudiada a nivel internacional. ${ }^{12}$

Como consecuencia del interés suscitado por estas dos propuestas (Parque de las Colonias y Parque Agrario) la Universidad Politécnica de Cataluña y el Massachusetts Institute of Technology, arrancan en 1999 una investigación conjunta sobre el planeamiento territorial basado en la revalorización de recursos patrimoniales, en la que analizan numerosas experiencias de parques patrimoniales en todo el mundo e intentan aplicar las lecciones aprendidas en el proyecto de un eje patrimonial del río Llobregat, que más adelante comentaremos. $^{13}$

\subsection{Una primera madurez: iniciativas de difusión de los paisajes del trabajo}

La labor de difusión ha estado protagonizada por diferentes departamentos universitarios, pero asimismo han tenido una importancia capital los cursos y publicaciones del INCUNA, los del Instituto del Patrimonio Histórico Español o

\footnotetext{
${ }^{12}$ Lo esencial de la propuesta se recoge en una quincena de publicaciones en diferentes países, entre otras:

"El Parque Agrario del Baix Llobregat" en Patrimonio y proyecto territorial. Diputación de Barcelona. Barcelona, 2004

"European Precedents" en Designing the Llobregat Corridor. Cultural Landscape and Regional Development. Projectant l'eix del Llobregat. Paisatge cultural i desenvolupament regional.

"O Parque Agrícola do Baix Llobregat" en Espaço Público e a Interdisciplinaridade Centro Português Design. Lisboa, 2000.

"Acerca de los espacios públicos contemporáneos" en Marina Waisman número 3. Córdoba (Argentina), agosto 2000.

"El Parc Agrari del Baix Llobregat" en Àrea Revista de Debats Territorials nº 8. Barcelona, marzo 2000.

"Des critères pour construire la périphérie" en La Ville Émergente. Éditions de l'Aube. París, 1997.

Cabe citar además el boletín Notícies del Parc Agrari del Baix Llobregat que se publica periódicamente.

${ }_{13}$ El resultado de estos trabajos se recoge en el libro antes mencionado Designing the Llobregat Corridor.

Cultural Landscape and Regional Development.
} 
los cuadernos del Instituto Andaluz del Patrimonio Histórico. Hay que destacar la labor pionera y de extraordinaria relevancia y originalidad a nivel internacional del Sistema del Museo de la Ciencia y de la Técnica de Cataluña. Surge en 1983 y bien pronto manifiesta su voluntad de constituir un museo nacional de la industrialización, atento a la singular diversidad de los paisajes industriales catalanes.

Esto le lleva, desde una sede única donde conservar colecciones de objetos técnicos, a extenderse por todo el territorio, intentando formar museografías de cada una de las manifestaciones de la especialización industrial en Cataluña (tornería, piel, papel, cemento, aguardiente, carbón, energía hidráulica, corcho, algodón, estampado textil, harineras, ferrocarril...). Hoy en día forman parte del Sistema una veintena de museos y otras diez estructuras de la industrialización han presentado su candidatura con la voluntad de integrarse en el mismo. Desde cada uno de ellos se desarrolla una política de estudio, inventario, conservación y restauración de bienes muebles e inmuebles.

El aspecto más destacable de estos museos es su funcionamiento como sistema, de manera que cada elemento se vincula a un fragmento del territorio y trata de explicar una parte de la historia de la industrialización. Para ello se desarrollan programas diversos, coordinados bajo una única imagen institucional. Y lo hace estimulado por la variedad de un paisaje que, transitando de la montaña al llano y al mar, desde un norte húmedo al sur seco, se caracteriza por una base geológica que ha dado lugar a explotaciones minerales diversas; por pequeños desniveles que permiten aprovechar la fuerza del agua, y por una rica diversidad productiva a lo largo de la historia. Esto contrasta con numerosas regiones europeas, de marcada especialización industrial.

Considero que una vez alcanzada la madurez y el reconocimiento internacional, el sistema debería plantearse nuevos retos, como el de dar a conocer el entorno de cada una de sus sedes, especialmente la incidencia de 
cada actividad productiva en su territorio, integrando los paisajes culturales como nodos de la red de museos y lugares patrimoniales. Un mecanismo para llevar esto a cabo es la definición de itinerarios temáticos a lo largo de diferentes paisajes culturales: áreas industriales papeleras, cuencas textiles laneras, algodoneras o de hilaturas, zonas mineras, áreas de aprovechamiento hidráulico-hidroeléctrico, etcétera.

\subsection{Marco legal y administrativo}

Desde el punto de vista normativo la consideración del paisaje del trabajo como un testimonio material de nuestra cultura es un fenómeno reciente. El primer texto relevante es la Ley de Patrimonio Histórico Español, de 1985, que no le reconoce una significación y tratamiento específicos. Aún así constituye un avance significativo que la noción de patrimonio se amplíe a toda expresión de la cultura material y testimonio de civilización.

En cambio, en el marco normativo autonómico el patrimonio industrial y los paisajes del trabajo encuentran, por fin, un tratamiento específico, que llega a incluir la dimensión territorial. No todas las leyes autonómicas nos ofrecen un perfil común: algunas consideran dicho patrimonio como parte del arqueológico, como la pionera ley de Castilla-La Mancha de 1990; en otras está integrado en el etnográfico, como en la de Madrid de 1998; un tercer grupo de textos se refiere al patrimonio industrial como arqueológico y etnográfico al mismo tiempo, caso de la Ley de Galicia de 1995. Más interesante es la Ley de Cantabria de 1998, que introduce una nueva referencia a "los espacios industriales y mineros"; o la del Principado de Asturias en 2001, donde se individualiza el patrimonio industrial. 


\section{INTERVENCIONES SOBRE EL PATRIMONIO DEL TRABAJO}

\subsection{Primeros instrumentos de intervención: una actitud preventiva}

El creciente interés del sector universitario por el patrimonio industrial se manifiesta igualmente en iniciativas como la elaboración de inventarios y catálogos. Aunque encontramos antecedentes interesantes en el libro de César Martinell sobre construcciones agrarias, cabe reconocer como pionero el Inventario del Patrimonio Industrial Histórico de Asturias (1987) del Departamento de Geografía de la Universidad de Oviedo, en colaboración con la Consejería de Cultura del Principado. Y a éste seguirían otros muchos hasta nuestros días, que tienen el indudable mérito de reclamar para aquellos paisajes del trabajo su condición de recursos patrimoniales.

Precisamente pocos años después se lleva a cabo el inventario de recursos patrimoniales de la cuenca del río Llobregat. Dicho documento supone un avance cualitativo, porque a partir de la identificación de los recursos más relevantes pretende ofrecer una interpretación estructurada y atractiva de los mismos, narrar una historia, capaz de atraer visitas e inversiones, de descubrir oportunidades de actividad y áreas de proyecto, de situar el territorio en condiciones de iniciar un nuevo impulso de desarrollo económico. ${ }^{14}$ Se trata en esencia de trascender el inventario y cohesionar los recursos culturales a partir de una idea-fuerza territorial, de dotarlos de estructura, de verificarla desde ensayos de proyectos, de construir una hipótesis de interpretación de un episodio relevante y de adelantar criterios para la ordenación de un territorio, para la gestión coherente de aquellos recursos.

La investigación parte de una hipótesis interpretativa: la cuenca del río Llobregat atraviesa y vertebra la provincia de Barcelona y su curso ha sido testigo de los principales episodios de la industrialización catalana. Se necesita

\footnotetext{
${ }^{14}$ D. Calatayud, J. Galindo, A. Giocoli, R. Jossa, D. Martínez, A. Lista, F. Rubio, J. Sabaté (coordinador) y P. Vall "Valoración de los recursos patrimoniales, de la estructura y ordenación del río Llobregat", Diputación de Barcelona, 1999.
} 
una historia que narrar, un hilo conductor. Por ello defiende que se trata seguramente del río más trabajador de Europa, por haber sido explotado durante siglos, prácticamente desde que nace, para mover molinos y turbinas, para alimentar poblaciones e industrias $y$, para crear pacientemente un delta agrícola, para ser embalsado y represado, y para acabar exhausto, y sin apenas caudal, en su desembocadura. Precisamente por todo ello concentra numerosos vestigios patrimoniales a lo largo de su cuenca. $Y$ así, siguiendo el curso del río se explica la historia del desarrollo industrial de Cataluña, identificando sucesivos episodios:

- Los excedentes de la rica huerta de Manresa, regada con aguas de una magnífica acequia de casi treinta kilómetros, construida en el siglo XIV, dan pie, con el transcurso del tiempo, a una primera industria urbana.

- Río arriba, ya a lo largo del siglo XIX, el agua empieza a mover las máquinas, y de los primeros molinos pasamos al extraordinario paisaje de las colonias textiles a partir de 1870.

- En la desembocadura del río, a mitad del siglo XIX, se crea un territorio agrario moderno con una esforzada construcción del delta.

- El aprovisionamiento de la ciudad da lugar a la aparición, cerca de los Pirineos, de fábricas de cemento y minas de carbón entre 1900 y 1920.

- Su transporte exige la instalación de líneas ferroviarias para salvar la distancia de la montaña al llano hacia 1930.

- Y también se genera una incipiente industria turística en el entorno de Montserrat, montaña sagrada de Cataluña, con la consecuente construcción de nuevos accesos (ferrocarril, funicular, aéreos y carrilet).

- Finalmente, la regulación del río, la construcción en 1970 de un pantano, y las extracciones y vertidos de una industria moderna, que obedece a nuevos patrones de localización, dan paso a la decadencia del sistema y, al mismo tiempo, a una creciente crisis ambiental. 
Los márgenes del río más trabajador de Europa muestran claros síntomas de agotamiento. Numerosos vestigios de un pasado floreciente (puentes medievales, molinos, presas, canales y acequias, fábricas y colonias industriales, instalaciones mineras, ferrocarriles y funiculares...) están abandonados. Pero seguramente los testimonios de dicha decadencia pueden servir de base para un nuevo impulso.

En esto se basa el proyecto del Eje patrimonial del Llobregat. Y también en el entusiasmo de diversos agentes locales para lograr cohesionar los recursos patrimoniales (naturales y culturales) a partir de una idea-fuerza territorial. Se pretende dotar a cada área de estructura, verificarla desde ensayos propositivos, construir una hipótesis de interpretación de un episodio histórico relevante $y$, en el límite, avanzar criterios para la ordenación conjunta de la cuenca fluvial, para la gestión coherente de todos aquellos recursos. Hoy en día los Parques Patrimoniales del Carbón, de las Colonias Industriales, de la Acequia y el Parque Agrario, constituyen unos primeros frutos de un proceso aún en curso. Asistimos a la reactivación de una cuenca fluvial a partir de la puesta en valor de sus recursos patrimoniales.

\subsection{Intervenciones aisladas}

Si en una primera categoría incluimos los esfuerzos de reconocimiento y protección, otra segunda recoge numerosos ejemplos de recuperación de viejas fábricas o almacenes destinados a museos, centros de arte o lofts. Se trata por lo general de construcciones singulares, vinculadas a la memoria industrial, que han pasado por un largo período de abandono, han sido reconocidas después como bienes de interés cultural, y se han convertido finalmente en operaciones emblemáticas de renovación urbana.

En algunos casos se acondicionan edificios abandonados como modernos centros de empresa. Cabe citar el Centro Municipal de Empresas La Curtidora, 
en Avilés, o Cristasa, que ocupa el edificio de una antigua fábrica de cristal en el barrio gijonés de La Calzada, ambos localizados en Asturias. En Mallorca se han rehabilitado dos grandes fábricas de calzado, Can Pellers y Can Ferrer, en el centro histórico de Binissalem, ubicando en ellas empresas de informática, servicios avanzados y tele trabajo.

La lista de ejemplos asturianos es ya muy larga (museos del Ferrocarril en Gijón; de la Minería en El Entrego; de la Sidra en Nava; Etnográfico de Grandas de Salime; ecomuseo de Taramundi y Mazonovo). Como lo es en Cataluña, donde encontramos muchísimos casos dignos de mención (Depósito de las Aguas, Ca l'Arañó, La Sedeta, museos del SMCTC, Vapor Vell de Sants, Can Batlló, Tecla Sala, naves Vilumara en I'Hospitalet, estación de Francia, CaixaForum, Fabra y Coats, diversas cavas y bodegas restauradas, harinera de Castellón de Ampurias, fábrica de azúcar en Vic....).

La trascendencia de estas piezas recuperadas no es en absoluto desdeñable, aunque se trata de un tipo de intervenciones centradas exclusivamente en el objeto, en la recuperación las más de las veces de un hermoso contenedor, donde las huellas de la historia apenas son reivindicadas o aparecen como una mera excusa.

\subsection{Planes y programas}

En tal contexto, representa un notable avance el estructurar mediante diferentes programas públicos (nacionales o comunitarios) la conservación y rehabilitación del patrimonio industrial. Cabe destacar especialmente el Plan Nacional de Patrimonio Industrial vinculado al Instituto del Patrimonio Histórico Español que distingue tres tipos de bienes industriales:

- Elementos aislados, que son testimonio representativo de una actividad industrial. 
- Conjuntos en los que se conservan todos los componentes materiales y funcionales, y que constituyan una muestra coherente y completa de una determinada actividad.

- Paisajes industriales donde se conservan todos los componentes esenciales de los procesos de producción de una o varias actividades industriales interrelacionadas.

Este Plan incluye piezas desde mediados del siglo XVIII y tienen cabida en él todas las manifestaciones arquitectónicas o tecnológicas relacionadas con las actividades de producción y distribución. A los restos muebles e inmuebles de la industrialización se suman fuentes documentales escritas, gráficas y orales.

Cabe hacer referencia asimismo a otras diversas operaciones que se han abordado desde una perspectiva más incluyente y estructurada (en Andalucía a partir del soporte del IAPH, en Asturias gracias a la labor del Incuna y en Cataluña a través del Sistema de Museos de la Ciencia y la Técnica).

\subsection{Proyectos territoriales}

En una última categoría podemos recoger algunos ejemplos recientes que ilustran la identificación entre patrimonio industrial y revalorización del territorio. Por ejemplo tenemos en Asturias los Itinerarios de patrimonio industrial, cultural y natural.

A otra escala encontramos la red de parques mineros españoles que se ha ido tejiendo en los últimos años. Supone la reconversión de espacios industriales abandonados en espacios de ocio y recreo, con un componente de turismo cultural importante. Son ámbitos al aire libre, creados a partir de explotaciones históricas, un singular y mal conocido patrimonio que hoy forma parte de la oferta turística de numerosas comunidades autónomas. 
Los ejemplos mejor conocidos son expresivos de un paisaje modelado por el hombre en su afán de extraer las riquezas del subsuelo y prueban la nueva dimensión adquirida por el patrimonio industrial como recurso económico y cultural. Entre éstos podemos citar: el parque arqueológico de Las Médulas (León), antiguas exploraciones auríferas de la época de los romanos declaradas recientemente Patrimonio de la Humanidad; las minas de plomo y plata de la Sierra de La Unión (Murcia); las de plata de Hiendelancina (Guadalajara); las de sal potásica de Cardona (Barcelona) o el Parque Temático Minero de Río Tinto (Huelva). Asimismo se consolidan proyectos como el Parque Minero-industrial de Linares, en Jaén; el Museo Regional de la Minería y la Siderurgia de Castilla y León en Sabero, León; o el Museo de la Minería de Puertollano, en Ciudad Real.

Cabe finalmente detenerse en algunos proyectos territoriales donde el patrimonio industrial es el verdadero protagonista, tales como los planes directores urbanísticos de las colonias industriales del Llobregat y del patrimonio industrial del Ter y Freser.

Antes de alcanzar el llano, el río Llobregat corre encajado dentro de un valle desdibujado. Desde mediados del siglo XIX este espacio es escenario de una transformación sorprendente, de la mano de algunas industrias que buscan la fuerza del agua y que, en ocasiones, huían del ambiente conflictivo de las ciudades.

Las fábricas crecen aprovechando cada meandro, pero nunca solas. Alrededor suyo, los mismos empresarios construyen pequeños asentamientos urbanos, siempre ordenados y a veces modélicos, donde los trabajadores viven a la sombra de la fábrica a lo largo de diversas generaciones. El resultado es un paisaje singular, mitad industrial y mitad agrario, habitado por obreros que a su vez eran agricultores. Es una sucesión de quince ciudades en miniatura, donde siempre están presentes la fábrica, la iglesia, las casas de los trabajadores, el 
canal que aporta las aguas, los huertos cerca del río y la villa de los propietarios.

A veces, la estructura es más compleja y aparecen un teatro, una escuela y una gran plaza pública. Algunas de estas colonias industriales resultan tan interesantes que para convertirlas en museo no ha hecho falta más que colocar las indicaciones. Las calles, hoy silenciosas, tienen el carácter de las colonias centroeuropeas y de las primeras ciudades-jardín inglesas. Los alrededores, en cambio, son todavía el reino de las casas de labranza, a veces monumentales, y de los campos que aprovechan los amplios márgenes bajo pequeños cerros todavía boscosos.

Pero con el tiempo los cambios en los mercados no dejan espacio para estas particulares formas de industrialización dispersa. La electricidad permite poner las fábricas allá donde se quiere y la economía global nos enseña que la concentración es la fuerza. Las fábricas de las viejas colonias ya no funcionan y muchos campos de alrededor tampoco se trabajan. El trabajo se fue y con él los habitantes de esta ciudad discontinua que creció allí, donde un salto permitía aprovechar la fuerza del agua.

Sobre este ámbito se habían venido desarrollando bien diversas iniciativas que culminaron con la constitución de un Consorcio del Parque Fluvial del Llobregat $^{15}$ en 2003 con el objetivo de impulsar la actividad turística y en general la promoción económica; preservar y difundir el patrimonio cultural y natural y coordinar la gestión supramuncipal y el planeamiento conjunto del parque.

Entre 2005 y 2007 se elabora un Plan Director Urbanístico al objeto de resolver notables desequilibrios y de preservar, revalorizar y estructurar el patrimonio industrial a lo largo del valle. Cabe destacar la naturaleza de dicha figura urbanística, a una escala intermedia entre lo urbano y lo territorial, con un

\footnotetext{
${ }^{15}$ Formado por la Generalitat de Catalunya, la Diputación de Barcelona, el Consejo Comarcal de Berguedà y diversas entidades representativas de la sociedad civil local.
} 
ámbito geográfico unitario que permite la comprensión integral del paisaje fluvial, al tiempo que la regulación detallada de cada colonia.

La situación de partida es dramática: los habitantes de las colonias parecen ciudadanos de segunda, sin transporte público, ni servicios urbanos o dotaciones básicas. Las fábricas cierran y los residentes van marchando, y como resultado encontramos una estructura productiva envejecida. La convivencia entre industria y residencia se hace difícil; no existen casi espacios libres públicos y la estructura urbana resulta insuficiente.

Para reactivar este patrimonio resulta imprescindible asegurar condiciones de prosperidad económica y de calidad de vida. Una colonia viva requiere una fábrica viva y, para ello, promover usos de valor añadido y activar dinámicas económicas, turísticas y residenciales, aceptando incluso pequeños crecimientos y cambios de uso industrial a terciario o residencial. Convertir las colonias en barrios equipados implica atraer habitantes, aceptar que puedan crecer hasta una dimensión que permita reclamar servicios y transporte público. Frente a la escasa dinámica demográfica y económica, al deterioro del patrimonio, a la escasez de recursos..., se busca un balance entre conservación y transformación, ofreciendo un pacto viable a los intereses públicos y privados.

Se vincula la mejora urbana de las colonias a su promoción económica, a los sectores que puedan generar riqueza y reinvertirla. $Y$ esto lleva a definir unas reglas precisas de crecimiento, unos criterios compositivos basados en un riguroso análisis. Y lleva asimismo a diseñar procesos de reactivación fundamentados en el turismo cultural y en atraer a las viejas fábricas nuevas actividades económicas.

El Plan Director Urbanístico se plantea como objetivos:

- Convertir las colonias en barrios dignos (calidad de los servicios, urbanización y transporte público). 
- Preservar sus valores patrimoniales, con pragmatismo y sensibilidad.

- Entender que reforma urbana y promoción económica, son dos caras de la misma moneda, entender la necesidad del impulso privado en un territorio extenso, periférico y con escasa propiedad pública.

- Para ello actúa a la vez sobre dinámicas industriales, turísticas y residenciales:

- Impulsando la plena ocupación del suelo industrial compatible con uso residencial.

- Mejorando los factores localización (acceso, agua, energía, telecomunicaciones...).

- Promoviendo usos de mayor valor añadido (incluso propiciando el cambio de uso industrial a terciario o, incluso, residencial.

- Generando plusvalías que han de revertir en consolidar y mantener espacios libres y compensar el sobrecoste del patrimonio.

- Impulsando dinámicas empresariales basadas en el turismo cultural (museos, centros de interpretación, ruta de las colonias,...).

- Atrayendo población, y en lo posible joven, básica para justificar transporte público, infraestructuras, servicios y dotaciones (equipamientos y espacios libres).

La mejora urbana de las colonias ha de permitir consolidar su condición de barrios integrados en una auténtica ciudad lineal:

- Rehabilitando edificios y espacios libres. Se identifican edificios de valor patrimonial y se elaboran directrices para su rehabilitación y adaptación a normativas de seguridad, accesibilidad y estándares de confort, así como pautas de ordenación de los espacios libres.

- Garantizando una buena convivencia de zonas residencial e industrial, promoviendo usos productivos limpios y compatibles y la segregación de accesos industriales y residenciales; evitando la fragmentación de la propiedad de la fábrica y transformaciones poco respetuosas; promoviendo en su caso la transformación a usos comerciales, terciarios o residenciales. 
- Dotando a las colonias de los servicios urbanos necesarios (limpieza espacios libres; atención médica domiciliaria; recogida residuos; tienda de barrio; saneamiento; transporte público.

\section{CONCLUSIONES}

Los paisajes del trabajo están teniendo una creciente importancia en el desarrollo económico regional de base local. Pero esto no debe interpretarse como el final de un recorrido. Dichos paisajes pueden llegar a constituir un vehículo, un eficaz instrumento para alcanzar el objetivo de construir entornos más diversos y cargados de identidad; y para reactivar determinados territorios.

Una de las propuestas más ambiciosas y relevantes en esta línea es la Belvedere Nota holandesa, aprobada en 1999, e integrada en el $5^{\circ}$ Documento de planeamiento físico de aquel país. En ella se pretende utilizar los recursos culturales para mejorar la calidad de los ambientes urbanos y rurales, incorporando la identidad histórico-cultural dentro de los procesos de planeamiento.

La selección en todo el país de diversas áreas Belvedere (que en base a criterios arquitectónicos, históricos o arqueológicos incorporan a veces ciudades enteras y paisajes extensos); la definición de proyectos estratégicos en las mismas; la voluntad de trabajar desde lo local, diseñando proyectos y actuaciones de cooperación y consenso; la protección mediante la transformación, superando posiciones conservacionistas, pretende en definitiva fundir la historia cultural, con origen en el pasado, con el planeamiento, con voluntad de proyectar el futuro.

En este sentido creemos que deben orientarse los esfuerzos: en situar el paisaje como eje central de los instrumentos y planes de ordenación. Paisaje en su más amplio sentido, natural y cultural; paisaje no como resultado acabado de unos procesos, sino como realidad en continuo cambio; paisaje y 
territorio no como mero soporte, sino como factor de transformación. Y en esta línea los paisajes del trabajo están llamados a jugar un papel relevante, porque constituyen la expresión de la memoria, de la identidad del lugar.

La proliferación de estudios, proyectos y realizaciones en España durante estos últimos años se ha producido aún a pesar de la falta de un reconocimiento institucional suficiente; de la escasez de estudios de base; de la debilidad y amenazas que se ciernen sobre el patrimonio heredado, y máxime del industrial; del insuficiente apoyo económico, programático y administrativo, más aún si lo comparamos la merecida atención que ha ido conquistando el paisaje natural.

Desde el punto de vista legislativo dicha demanda debe traducirse en un reconocimiento y defensa expresa de los paisajes culturales, ya sea desde las Leyes de Paisaje, o de un instrumento propio, como se ha ensayado en el caso holandés, o en los de Aragón y Asturias. Desde el punto de vista instrumental es imprescindible impulsar estudios, como los que se han realizado relativos a los paisajes naturales (y agrícolas), de reconocimiento e inventario, de catalogación y de medidas de intervención. ${ }^{16}$

Quizás la figura más adecuada podría ser un Libro Blanco de los principales paisajes del trabajo por autonomías, atendiendo a su representatividad, estado de conservación, amenazas, alternativas de tratamiento..., con el objetivo final de llegar a disponer de una herramienta equiparable a los ya bien conocidos Planes de Espacios de Interés Natural, en defensa de la valorización de la rica biodiversidad patrimonial que ha informado e informa la evolución de este país. El reto sería disponer de unos Planes de Espacios de Interés Cultural (PEIC).

Desde el punto de vista del planeamiento y ordenación territorial, además de los mencionados PEIC, debe reconocerse el cometido de los paisajes culturales en el planeamiento urbanístico y territorial. En el límite cabe reclamar

${ }^{16}$ De manera cautelar y transitoria quizás convendría establecer medidas de protección de determinados ámbitos patrimoniales, que aseguren la defensa de aquellos recursos más amenazados en tanto no se desarrollen políticas y proyectos de revalorización. 
el protagonismo de determinados sistemas de recursos culturales como herramienta de revalorización e impulso económico de diversos territorios. Los planes territoriales pueden constituir en este sentido una figura idónea, al integrar una visión evolutiva de estos paisajes.

Finalmente la difusión y el aprecio por este legado patrimonial debe seguir incentivándose desde programas de formación (seminarios y congresos, cursos académicos...) y proyectos piloto, mediante campañas de divulgación y mentalización, y a través de proyectos de señalización homogéneos e integrales.

No es tan solo una cuestión de mero mantenimiento de un legado patrimonial, que ya sería importante en sí misma. Hoy más que nunca frente a la globalización y banalización de tantos paisajes debemos apostar por intervenir en ellos conservando su identidad, valorando su código genético, su memoria. Porque en la identidad del territorio está la alternativa de progreso y desarrollo de la sociedad que lo conforma. 